\title{
LES FIBRES MÉDULLEES DANS LA TOISON DE LA RACE OVINE DE TEXEL
}

\author{
PAR \\ J. ROUGEOT ( ${ }^{1}$ ) \\ Station de Recherches de Physiologie Animale \\ Centre National de Recherches Zootechniques, Jouy-en-Josas (S.-et-O.).
}

Chez les Moutons de la race de 'Texel, la toison recouvre entièrement le corps, ne laissant dégagées que la tête et la partie inférieure des membres. Les mèches composant la toison sont très longues ( $\mathrm{I} 4-\mathrm{I} 8 \mathrm{~cm}$ ) s'individualisant en petites pointes à l'extérieur. Les fibres sont en général assez grossières : elles sont classées dans les no 3 et 4 croisés $(29,5 \mu$ à $32,5 \mu)$. Cette toison importante protège efficacement l'animal contre les intempéries et permet l'élevage des Texel en plein air, même par les grands froids et la neige. I,e poids de la toison (4 à $5 \mathrm{~kg}$ chez les brebis, 5 à $6 \mathrm{~kg}$ chez les béliers) est une source de revenu non négligeable pour l'éleveur, d'autant plus que le rendement en lavé à fond est élevé (50 à $60 \mathrm{p}$. Ioo).

Un examen macroscopique préalable des toisons Texel nous a montré que celles-ci sont composées de trois types de fibres toutes très longues : la laine, l'hétérotype et le poil. Le iarre est totalement absent (P1. I et Pl. II).

Il nous a donc semblé intéressant d'essayer de préciser les proportions selon lesquelles se répartissent, dans la toison de cette race, les divers types de fibres et d'en déduire les conséquences possibles pour la sélection lainière.

Nous avons examiné ainsi 183 brebis Texel âgées de $I$ à 5 ans, et appartenant à trois excellents troupeaux.

Les prélèvements ont été faits à la fin du mois de février : à cette époque, les hétérotypes ont en effet à peu près atteint leur longueur maximale et se distinguent plus aisément des poils. On ne peut d'ailleurs reculer la date des prélèvements, car dès cette époque, une proportion appréciable d'hétérotypes ont cessé de croître et nous avons même trouvé quelques toisons où des hétérotypes commençaient à tomber.

Les observations ont été faites sur des mèches prélevées sur le milieu du côté de l'animal, au niveau de la dernière côte. Trois échantillons, de Ioo à I5o fibres chacun, furent ensuite prélevés au hasard, dans chaque mèche et toutes ces fibres furent examinées une à une dans l'orthodi-

(1) Avec la collaboration technique de Jacqueline Mergelsberg. 
chlorobenzène : les brins de laine constitués entièrement de kératine dont l'indice de réfraction est le même que celui de l'orthodichlorobenzène, deviennent invisibles dès qu'ils sont immergés dans ce liquide. Au contraire, les poils et les hétérotypes demeurent visibles grâce à leur canal médullaire qui, avec ses inclusions d'air, présente un indice de réfraction très différent de l'orthodichlorobenzène, les poils se distinguant des hétérotypes, par leur canal médullaire continu, s'étendant d'un bout à l'autre de la fibre : en effet, chéz les hétérotypes le canal médullaire n'existe que dans la partie distale de la fibre et occupe une proportion très variable de la longueur de la fibre (P1. III).

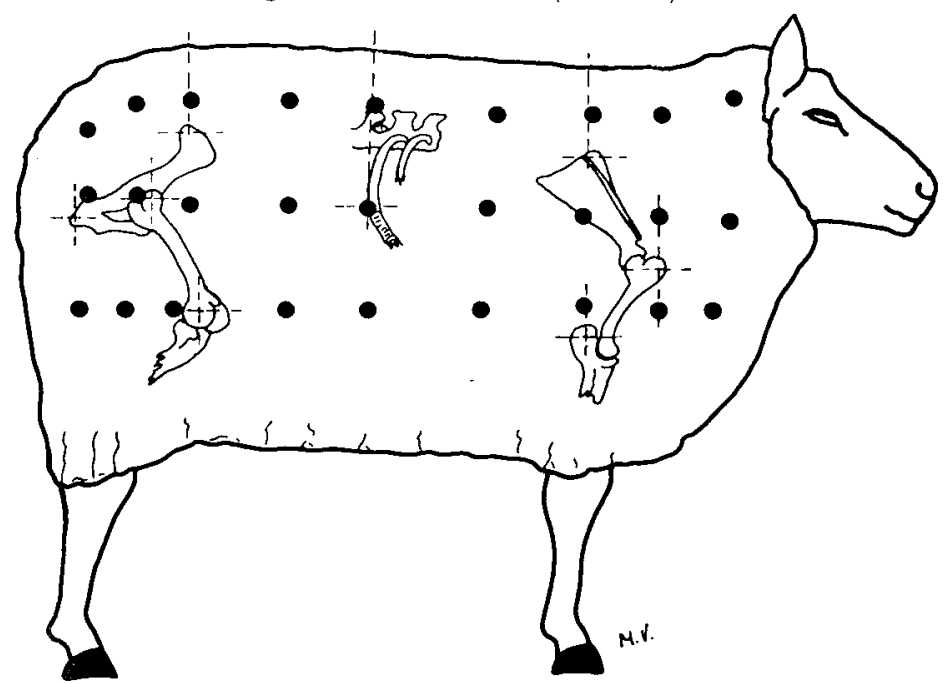

Fig. I. - Les points indiquent les lieux de prélèvements ; 9 le long de la colonne vertébrale ; 6 dans la région postérieure ; 6 sur le côté ; 6 dans la région antérieure. -- Les points de rencontre des traits discontinus indiquent les repères osseux utilisés : $I^{\circ}$ 'Tubérosité ischiatique (pointe de la fesse) ; Épine iliaque antérieure et supérieure (hanche): Dernière côte; Partie supérieure de l'épine acromienne (omoplate). - $2^{\circ}$ Articulations : coxo-fémorale (hanche); fémoro-tibiale-rotulienne (cuisse-jambe); scapulo-humérale (épaule); radio-cubitale (coude).

Cette méthode nous a permis d'étudier l'état de médullation des mèches aux points de vue quantitatif et qualitatif :

Io Dans l'analyse quantitative de l'état de médullation de la mèche, nous avons déterminé le rapport du nombre de fibres médullées poils et hétérotypes, au nombre de brins de laine dans chaque mèche.

$2^{\circ}$ Dans 1'analyse qualitative de la médullation, nous avons étudié la répartition de la médullation sur la longueur de la mèche.

\section{I. - Analyse quantitative de l'état de médullation de la mèche}

Les résultats de l'analyse sont exprimés dans le tableau I. Nous voyons immédiatement que les Texel présentent dans la mèche située sur le côté, des pourcentages de laine très variables. Ainsi, 3,8 p. roo 
d'entre eux n'ont que de la laine à cet endroit, tandis que 2,7 p. Ioo n'en ont point du tout. En groupant les deux premières et les deux dernières colonnes du tableau I, ce qui est tout à fait légitime, on voit que la répartition des animaux entre les 5 classes ainsi obtenues est très uniforme.

TABLEAU I

\begin{tabular}{|c|c|c|c|c|c|c|c|c|}
\hline & I & I & 3 & 4 & 5 & 6 & 7 & Total \\
\hline $\begin{array}{l}\text { Pourcentage de fibres médul- } \\
\text { lées dans la méche prélevée } \\
\text { au flanc............... }\end{array}$ & $0 \%$ & $I-20$ & $2 \mathrm{I}-40$ & $4 I-60$ & $60-80$ & $8 \mathbf{I}-99$ & $100 \%$ & \\
\hline Nombre de toisons $\ldots \ldots \ldots \ldots$ & 7 & 28 & 35 & $3^{6}$ & 39 & 33 & 5 & 183 \\
\hline \multirow[t]{2}{*}{$\begin{array}{l}\% \text { des toisons dans chaque } \\
\text { classe par rapport au total. }\end{array}$} & 3,8 & ${ }^{2} 5,3$ & \multirow[t]{2}{*}{ I9,I } & \multirow[t]{2}{*}{ I9,7 } & \multirow[t]{2}{*}{$2 \mathrm{I}, 3$} & IS & 2,7 & \\
\hline & & & & & & \multicolumn{2}{|c|}{20,7} & \\
\hline
\end{tabular}

On peut donc dire que les fibres médullées se rencontrent en toutes proportions dans les mèches situées sur le flanc.

Si les mèches avaient été prélevées à l'épaule, nous aurions obtenu des échantillons ayant un pourcentage plus élevé de brins de laine. L'inverse se serait produit en faisant les prélèvements à la cuils: En effet, chez le mouton, le pourcentage des fibres médullées est plus élevé à la cuisse qu'au flanc, et plus élevé au flanc qu'à 1'épaule (W: LsoN, I929; ELPHICK, I932; LANG, I942).

Ce fait a été vérifié chez les Texel : l'analyse topographique de la répartition des fibres médullées a été effectuée à partir de I 8 mèches prélevées sur le dos et le côté droit de I2 Brebis : la figure I montre la localisation de ces prélèvements par rapport à des repères osseux commodes. L,es observations n'ont porté que sur un seul côté, étant donné que le plan de symétrie de l'animal est également un plan de symétrie en ce qui concerne la répartition des fibres médullées de la toison (ErPHICK, I932).

Dans chaque mèche, trois échantillons de $x 50$ à 200 fibres furent prélevés au hasard et leurs fibres examinées une à une dans l'orthodichlorobenzène.

Ces examens ont montré que dans la toison, le taux de fibres médullées varie suivant deux directions bien définies :

Io Une direction antéro-postérieure, le taux de fibres médullées allant en augmentant de la tête à la queue.

$2^{\circ}$ Une direction dorso-ventrale, la proportion des fibres médullées allant en augmentant de la région dorsale à la région ventrale.

Ainsi, c'est la partie inférieure et postérieure de la cuisse qui présente la proportion la plus élevée de fibres médullées tandis que c'est la région 

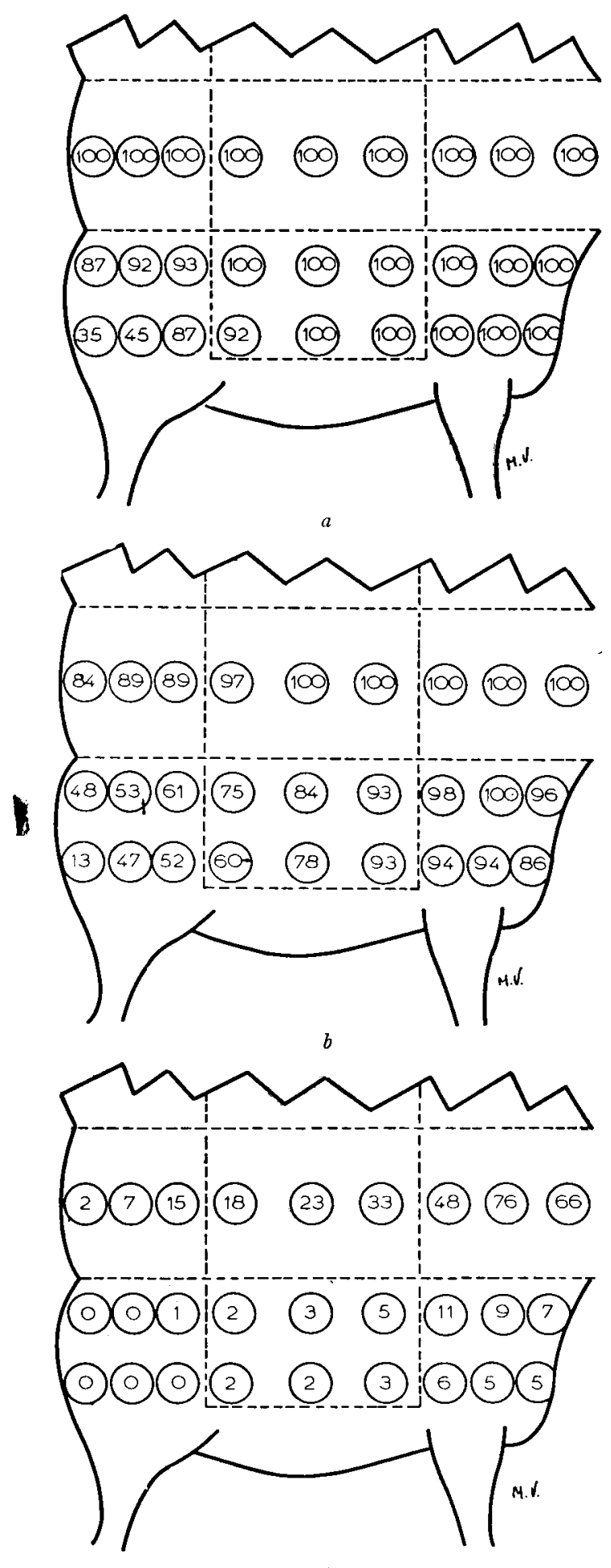

Fig. 2. - Exemples de la répartition des fibres médullées dans la toison des Texel : $a$ ) Type de toison présentant uniquement des brins de laine au flanc $($ Iо0 \%); $b$ ) Type de toison présentant une proportion assez élevée de laine au flanc $(84 \%)$; c) Type de toison présentant une très faible proportion de laine au flanc $(3 \%)$. 

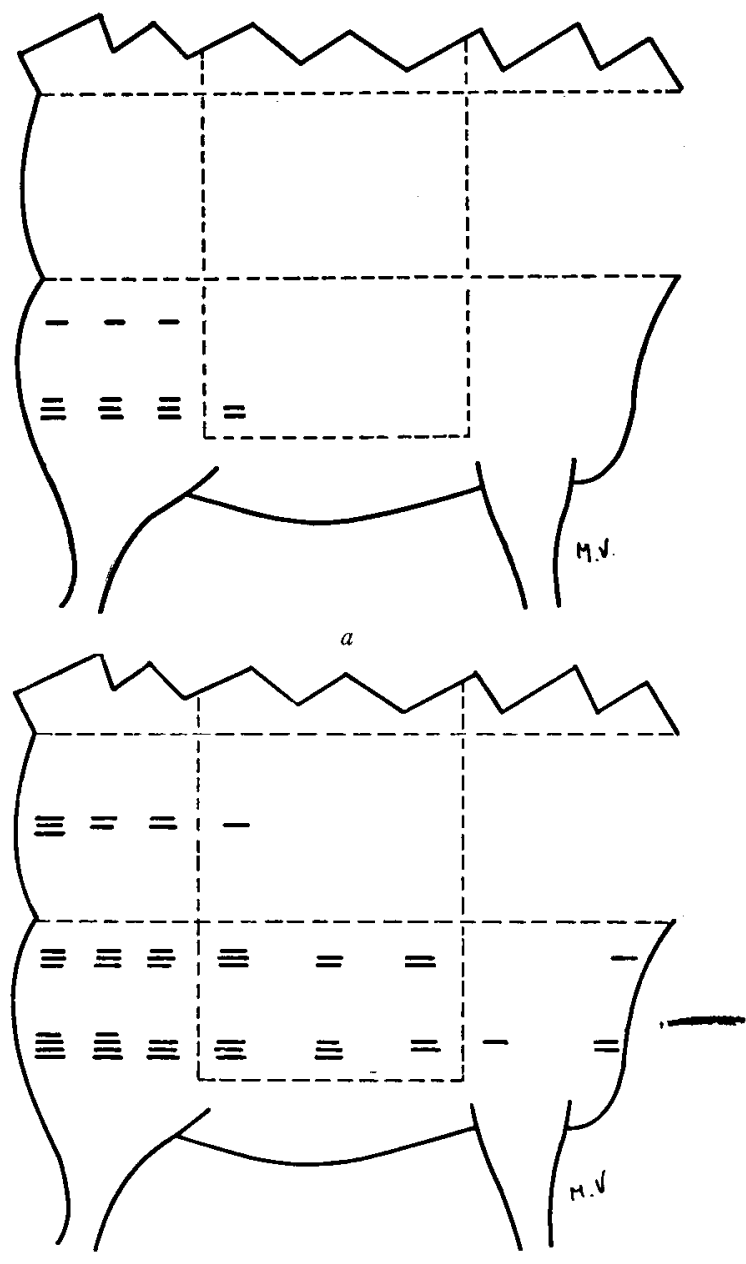

b

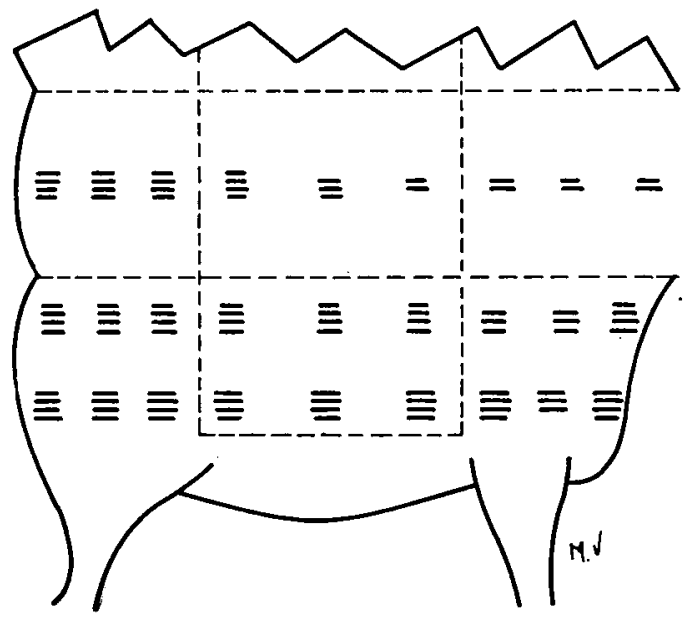

$c$

FIG. 3. - Proportion de la longueur de la mèche occupée par la médullation suivant l'emplacement sur la toison. Les trois exemples de toison $a, b, c$, de cette figure sont respectivement les mêmes que les exemples $a, b, c$, de la figure 2 , les mesures étant faites sur les mêmes mèches. Chaque trait signifie que la médullation s'étend sur un quart de longueur de mèche. 
du garrot et la partie supérieure de l'épaule qui présente le taux le plus élevé de brins de laine. On doit toutefois noter que la proportion de fibres médullées est plus élevée dans la région du poitrail et de la gorge que dans celle de l'épaule et du bras (fig. 2).

Le prélèvement effectué au flanc est donc très représentatif pour la détermination des proportions relatives des différents types de fibres de la toison.

\section{II. - Analyse qualitative de l'état de médullation}

Ia présence de poils, c'est-à-dire de fibres dont le canal médullaire s'étend sans discontinuité d'une extrémité à l'autre de la fibre a été reconnue dans une grande partie de mèches prélevées au flanc des animaux: en effet, en se rapportant au tableau II, on voit que sur I83 toisons examinées, 75, soit 4I p. Ioo, présentent des poils dans les mèches prélevées au flanc.

De plus, on constate que les mèches qui présentent des poils se rencontrent surtout parmi celles dont les proportions en fibres médullées sont élevées : ainsi $76 \mathrm{p}$. Ioo des mèches qui ont $8 \mathrm{I}$ à Ioo p. Ioo de fibres médullées possèdent des poils, tandis qu'on en trouve seulement II p. Ioo

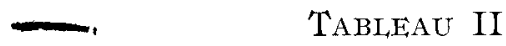

Répartition des toisons dont les mèches prélevées au flanc présentent des poils, suivant la proportion des fibres médullées de ces mèches.

\begin{tabular}{|c|c|c|c|c|c|c|}
\hline $\begin{array}{c}\text { Pourcentage de fibres médullées (poils et hétéro- } \\
\text { types) dans les méches prélevées au flanc, } \\
\text { par rapport au nombre total de fibres }\end{array}$ & $\begin{array}{c}\text { jusqu'à } \\
20 \%\end{array}$ & $2 I-40$ & $4 I-60$ & $6 \mathrm{I}-80$ & $8 \pi-100$ & Total \\
\hline Nombre de toisons examinées........... & 35 & 35 & $3^{6}$ & 39 & 38 & I 83 \\
\hline $\begin{array}{l}\text { Nombre de toisons présentant des poils dans les } \\
\text { mèches du flanc } \ldots \ldots \ldots \ldots \ldots \ldots \ldots \ldots \ldots \ldots \ldots \ldots\end{array}$ & 3 & 35 & 8 & 23 & 29 & 75 \\
\hline $\begin{array}{l}\text { Pourcentage des toisons présentant des poils } \\
\text { dans les mèches du flanc par rapport au nombre } \\
\text { total de toisons dans chaque classe } \ldots . . . . . .\end{array}$ & $10,7 \%$ & $34,3 \%$ & $22,2 \%$ & $59 \%$ & $76,3 \%$ & $41 \%$ \\
\hline
\end{tabular}

parmi celles qui comptent moins de $20 \mathrm{p}$. Ioo de fibres médullées (tableau II).

La part qui revient aux poils dans la médullation des mèches du flanc est très variable suivant les toisons, mais généralement, moins de 60 p. Ioo des fibres médullées sont constituées par des poils et les mèches qui ont la plus grande partie de leurs fibres médullées sous forme de poils sont relativement rares : en effet, seulement 5 p. roo des mèches oì l'on trouve des poils, ont plus de $80 \mathrm{p}$. Ioo de leurs fibres médullées appartenant à ce type (tableau III). 
TABleau III

\begin{tabular}{|c|c|c|c|c|c|}
\hline $\begin{array}{l}\text { Pourcentage des poils, par } \\
\text { rapport à l'ensemble des } \\
\text { fibres médullées (poils }+ \\
\text { hétérotypes) } \ldots \ldots \ldots \ldots \ldots\end{array}$ & $\mathrm{I}-20 \%$ & $2 \mathrm{I}-40$ & $+1-60$ & $6 r-80$ & $8 \mathrm{I-100} \%$ \\
\hline $\begin{array}{l}\text { Répartition en pourcentages, } \\
\text { des mèches du flanc, pré- } \\
\text { sentant des poils. ......... }\end{array}$ & 27 & 28 & $3^{2}$ & 8 & 5 \\
\hline
\end{tabular}

Quant aux hétérotypes, ils sont en général médullés, sur une grande partie de leur longueur : en effet, parmi les toisons qui, au flanc, ne présentent comme fibres médullées que des hétérotypes, 66 p. roo des mèches sont médullées sur au moins les $3 / 4$ de leur longueur et $85 \mathrm{p}$. Ioo sur au moins $1 \mathrm{a} \mathrm{I} / 2$ de leur longueur (tableau IV).

I1 ressort de cette analyse, qu'en ce qui concerne les mèches situées sur le flanc, les poils constituent une fraction importante des fibres médullées et que les hétérotypes possèdent en général un canal médullaire assez long.

Nous avons étudié enfin la variation de la médullation sur la longueur

\section{TABLEAU IV}

Importance de la longueur du canal médullaire chez les hétérotypes : répartition des toisons, dont les mèches prélevées au flanc ne présentent que des hétérotypes ou de la laine, suivant la proportion de la longueur de ces mèches occupée par la médullation.

\begin{tabular}{|c|c|c|c|}
\hline $\begin{array}{l}\text { Proportion de la longueur de la mèche } \\
\text { occupée par la médulation. ........ }\end{array}$ & plus des $3 / 4$ & $3 / 4 \grave{a} \quad 1 / 2$ & $\mathbf{I} / 2$ et moins \\
\hline Nombre de toisons (гог) & $6 \bar{j}$ & r9 & I 5 \\
\hline Pourcentage de toisons. $\ldots \ldots \ldots \ldots$ & $66,3 \%$ & I8,8\% & \\
\hline
\end{tabular}

de la mèche en fonction de l'emplacement de celle-ci sur le corps de l'animal. Pour cela nous avons utilisé les mèches qui avaient servi à la précédente analyse topographique. Ces mèches ont été classées en 5 groupes définis par la fraction de la longueur de la mèche occupée par les canaux médullaires : mèches médullées sur toute leur longueur sur les $3 / 4$, la I/2 ou le I/4 de celle-ci, et mèches sans fibres médullées.

Nous avons constaté, comme précédemment, que la médullation occupait une fraction de la longueur de la mèche d'autant plus importante que la proportion de fibres médullées de la mèche était élevée. Les

Annales de Zootechnie. - $195^{6}$. 
trois schémas de toison de la figure 3 illustrent parfaitement le parallélisme des deux caractères. Ce qui confirme que le prélèvement effectué au flanc est très représentatif de l'état moyen de médullation de la toison.

L'ensemble de ces observations nous permet donc de conclure en disant que les toisons des moutons de la race Texel sont composées de poils, d'hétérotypes et de brins de laine en proportions relatives très variables selon les individus, que les proportions relatives de fibres médullées sont, dans la plupart des cas, très importantes, que la médullation s'étend en général sur la plus grande partie de la longueur de la mèche ; enfin, la présence du jarre n'a pas été observée dans la toison des animaux examinés.

\section{Discussion}

On sait, notamment d'après les travaux de DRY (I935-I955) que la présence de fibres médullées dépend surtout de facteurs héréditaires. Nous venons de voir d'autre part que les poils, hétérotypes et brins de laine se trouvent en des proportions relatives très variables dans la toison des 'Texel. Cela signifie qu'il est possible d'obtenir dans cette race, par le jeu de la sélection, des animaux présentant des toisons composées de fibres médullées et de laine en des proportions relatives dont on peut fixer à l'avance les limites. Il n'est même pas du tout exclu, de pouvoir entreprendre la sélection de Texel dont les toisons seraient composées presque entièrement de laine, les dernières fibres médullées se réfugiant aux niveaux postérieur et inférieur de la cuisse.

Ainsi, le choix d'un type défini de toison, compte tenu des difficultés inhérentes aux travaux de sélection, est guidé finalement par le mode de vie de l'animal et les débouchés commerciaux offerts aux différents types de toisons.

I,es Texel sont élevés en plein air la plus grande partie de l'année, leur abondante toison leur permet de résister aux intempéries, la pluie et la neige glissent sur les extrémités pointues des mèches formées par les poils et les hétérotypes, sans pénétrer à l'intérieur de la toison. On peut donc admettre que le type actuel de toison chez les Texel convient à ce mode d'élevage.

Les débouchés commerciaux offerts aux toisons des Texel dépendent des proportions généralement assez élevées de poils et surtout d'hétérotypes qui s'y trouvent. En effet les poils et la partie médullée de l'hétérotype, qui est identique à tous points de vue au poil, possèdent des propriétés assez différentes de la laine. Les poils sont grossiers, beaucoup moins souples que la laine, mais ils possèdent par contre plus de "ressort". En outre, la grossièreté et la raideur de ces fibres jointes à la structure particulière de leur cuticule, formée d'écailles peu saillantes en hexa- 
gones à peu près réguliers, s'opposant aux écailles coronales très saillantes de la laine, leur confèrent une aptitude au feutrage beaucoup moins marquée.

L'hétérotype présente évidemment les propriétés du poil dans sa partie distale, grossière et médullée, et celles de la laine dans sa partie proximale, fine et non médullée : les propriétés du poil ou de la laine sont plus ou moins accentuées chez l'hétérotype suivant l'importance de l'une ou l'autre de ces structures.

Les toisons de Texel ne peuvent évidemment convenir, en raison de la présence des poils et des hétérotypes, aux utilisations textiles " nobles ", telles que les tissus de laine peignée, la bonneterie fine (jersey), etc.

Par contre, ces toisons, grâce aux propriétés de ressort des poils et des hétérotypes, à la douceur de ces fibres, à leur aptitude au feutrage moins prononcée, sont appréciées, sinon recherchées, pour certains articles de laine à tricoter, les draps grossiers, les couvertures, les tapis, les matelas.

Signalons à ce propos que la demande mondiale de laine à tapis est très importante et que par exemple, les U. S. A. importent presque totalement la laine à tapis nécessaire à leur industrie. I1 est très intéressant à ce propos, de comparer l'analyse que nous venons de faire de la toison des Texel et l'opinion de Von Bergen et Mauersberger, dans l'American Wool Handbook (1948) sur les conditions que devrait remplir une laine à tapis idéale :

Io "On devrait y dénombrer au moins I5 p. roo de fibres hétérotypiques. Ces fibres hétérotypiques devraient présenter une épaisseur d'au moins $30 \mu$ et l'épaisseur des fibres ne devrait pas présenter de variation supérieure à I 5 p. Ioo. La longueur atteinte par les fibres au cours de la durée normale de la croissance (I2 mois) devrait être an moins de Io $\mathrm{cm}$ et la longueur des fibres ne devrait pas présenter de variation supérieure à 20 p. Ioo. "

$2^{0}$ "On ne devrait pas y compter plus de 2 p. Ioo de jarre."

$3^{\circ}$ "On ne devrait pas y dénombrer plus de 85 p. Ioo de brins de laine. »

"L'épaisseur des brins de laine ne devrait pas dépasser $25,4 \mu$, l'épaisseur des fibres présentant une variation inférieure à $25 \mathrm{p}$. Ioo. La longueur atteinte par les brins de laine au cours d'une durée de croissance normale ( $\mathrm{I} 2$ mois) devrait être de $\mathrm{Io} \mathrm{cm}$ au moins, et la longueur des fibres ne devrait pas présenter une variation de plus de $25 \mathrm{p}$. Ioo.

On voit donc que la toison des Texel présente toutes les caractéristiques d'une laine à tapis idéale, telle que la définissent VON BERGFN et MAUERSBERGER.

Ajoutons que les troupeaux de moutons Texel étant généralement 
petits, très dispersés, et élevés dans les régions riches et peuplées des herbages, les demandes locales de laine à matelas suffisent souvent à absorber la production des éleveurs, dans des conditions intéressantes. I1 semble donc très intéressant d'orienter la sélection des toisons vers le type défini par Von Bergen et MaUkrsberger, c'est-à-dire d'éliminer les animaux dont les toisons présentent des poils et des hétérotypes trop grossiers en se gardant bien, toutefois, de rechercher une finesse excessive des fibres. Enfin, et c'est peut-être le problème le plus important pour lui, l'éleveur a toujours intérêt à améliorer le poids net de la toison.

Ainsi les toisons des Texel fourniront une matière de premier choix pour l'industrie des tapis, sans perdre pour autant leurs débouchés traditionnels.

\section{Conclusion}

L'analyse des toisons de 183 brebis Texel nous a permis de montre1 que, selon les individus, la laine, les poils et les hétérotypes se trouvaient en proportions relatives très variables dans la toison et que l'échantillon prélevé au flanc pouvait être considéré comme très représentatif de l'état de médullation de la toison.

Ces résultats et les considérations relatives aux débouchés commerciaux des toisons des Texel et au mode de vie de cette race, nous conduisent aux conclusions suivantes pour la sélection lainière :

I $^{\circ} \mathrm{L}$ a présence en des proportions variables, mais généralement élevées, de poils et d'hétérotypes peu grossiers, confère à la toison des Texel, des propriétés qui la font apprécier et rechercher par de nombreuses industries. Il n'y a donc pas avantage à sélectionner des toisons laineuses qui concurrenceraient difficilement les toisons composées uniquement de fibres de laine des races Ile-de-Firance ou Berrichonne du Cher par exemple. Il ne faut pas oublier non plus que, si l'élimination des fibres médullées par sélection est possible, elle exige un travail considérable et d'autant plus difficile que les proportions de fibres médullées sont plus élevées.

$2^{0}$ Il est préférable d'éliminer les animaux présentant des toisons trop grossières, c'est-à-dire les toisons possédant un pourcentage d'hétérotypes et surtout de poils trop élevé, ainsi que les toisons présentant des fibres médullées grossières : de cette façon, les toisons Texel fourniront aussi bien d'excellente laine à tricoter qu'une matière première de choix pour les tapis.

$3^{\circ}$ Il est toujours avantageux et relativement facile de sélectionner des animaux portant des toisons lourdes (mèches longues, densité de fibre élevée).

Reçu pour publication le 16 jantier 1956. 


\section{RÉFÉRENCES BIBLIOGRAPHIQUES}

Bergen (W. von), Mauersberger (H. R.). - American Wool Handbook, $2^{\mathrm{e}}$ éd., Textile Book Publishers, Inc., New-York, I948.

DrY (F. W.). - Hairy fibres of the Romney sheep. Wellington : G. H. Loney, Govt. Printer, 48 pp., I935.

DRY (F. W.). - Multifactorial inheritance of halo-hair abundance in NewZealand Romney Sheep. Aust. J. Agric. Res., 6, 608-623, I955.

ErrPHICK (B. L.). - The detection and estimation of medullated fibres in the New-Zealand Romney fleeces. J. Text. Inst., 23, 367-85, I932.

LANG (W. R.). - Some aspect of the occurrence and significance of hairy fibres in Australian wools. Gordon Inst. Techn. (Geelong, Austr.), Publ. no 3, 27 pp., I942.

WilsoN (J. F.). - The medullated wool fiber, Hilgardia, 5, I35-I52, I929. 
PLANCHE I

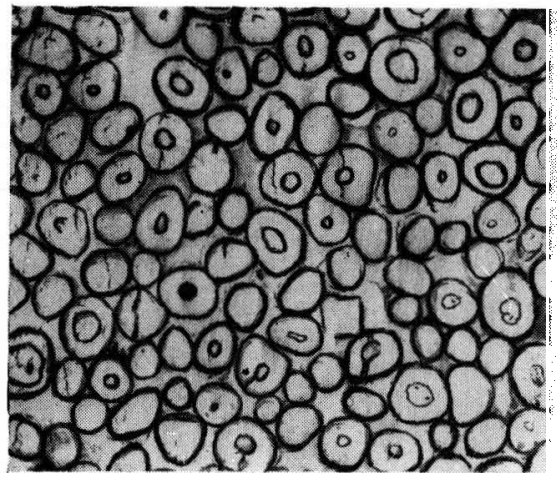

$a$

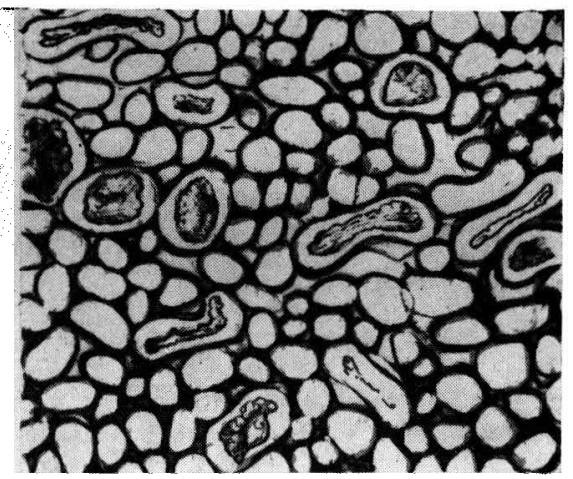

$b$

Fig. I. - Coupes transversales des miches de la toison du Mouton ( $\left.\times 8_{5}\right)$. a) Mèche de la toison de la race ovine de Texel. Mélange de laine, de poil et d'hétérotype. Les poils et les hétérotypes ont comme la laine une section droite à peu près circulaire. Les canaux médullaires présents seulement chez les poils et les hétérotypes, sont relativement fins; b) Mc̀che de toison de la race ovine Limousine. Mćlange de jarre et de laine. Les brins de jarre, très grossiers et présentant un canal médullaire très important, ont très souvent une section droite aplatie.

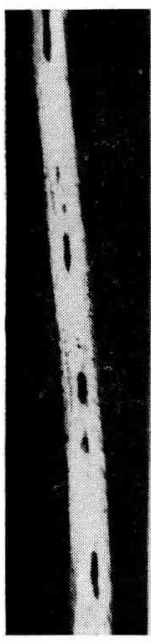

$a$

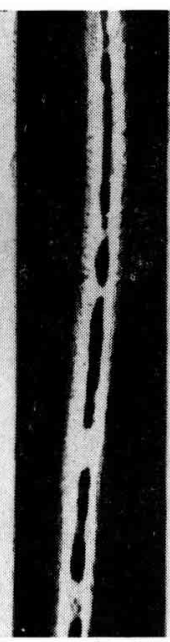

$b$

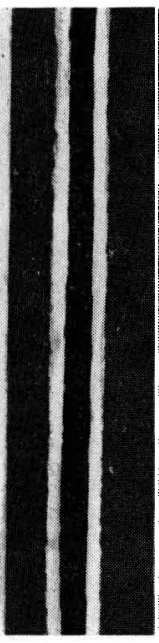

FIG. 2.

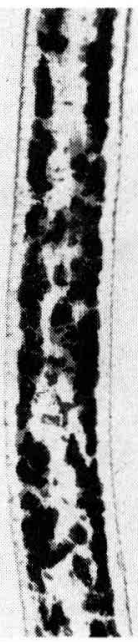

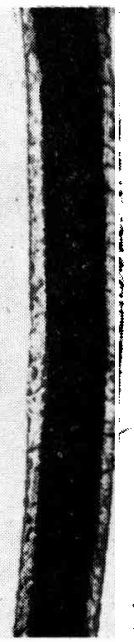

$b$

FIG. 3.

FIG. 2. - Canaux médullaires des poils $(\times 85)$ a) Canal mdéullaire fragmennaire; b) Canal médullaire interrompu; c) Canal médullaire continu. - Les canaux médullaires des poils (et des hétérotypes) étant relativement fins, ces fibres présentent des propriétés mécaniques intéressantes pour l'industrie.

FIG. 3. - Canaux médullaires du jarre $(\times 85)$. a) Jarre ayant une section droite aplatie, le canal médullaire forme deux bandes latérales; c) Jarre ayant une section droite onale ou circulaire. ... Les canaux médullaires constituant la majeure partie du jarre, ces fibres ne possèdent que de très médiocres propriétés mécaniques. En outre, la teinture des brins de jarre offre de grandes difficultés, dues principalement à la présence de l'air dans leurs énormes canaux médullaires. 


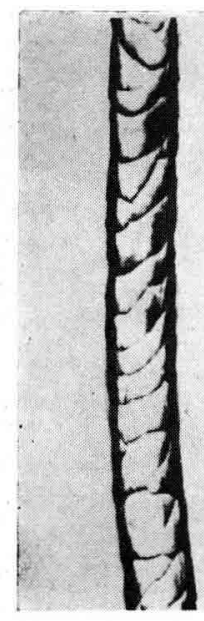

$a$

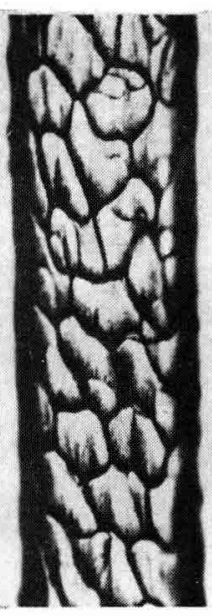

$b$

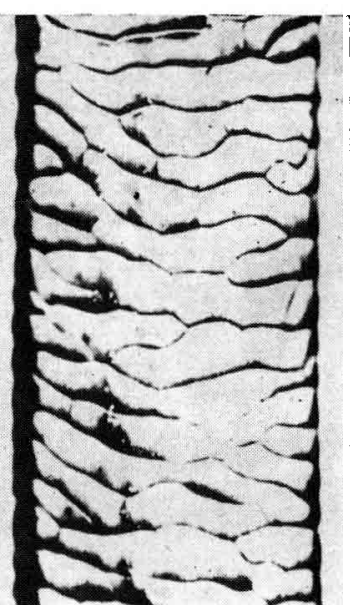

$c$

Empreinte des écailles des trois principaux types de fibres de la toison du Mouton. a) Brin de laine de Mérinos $(\times 300)$. Écailles coronales, très saillantes; b) Poil de Texel $(x$ 30o). Écailles en hexagones, jeu saillantes ; c) Jarre de Limousin $(x, 300)$. Écailles rectangulaires, peu saillantes. 
Planche III. - Détermination des poils et des hétérotypes dans une mèche de toison Texel par immersion dans l'orthodichlorobenzine.

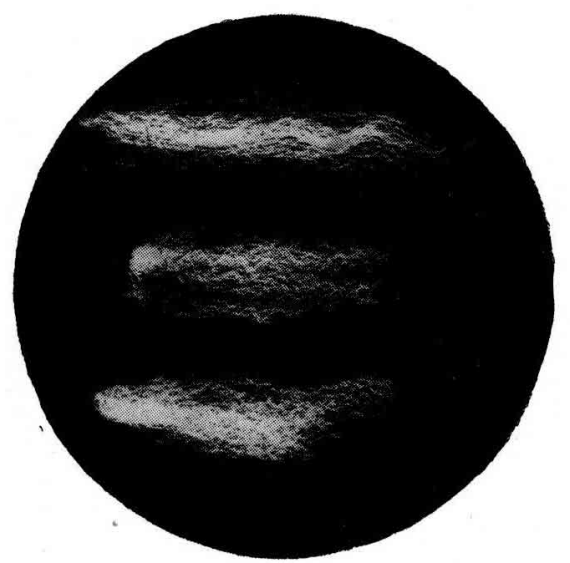

FiG. I. - Photographie de trois mèches avant immersion : Mèche supérieure: prélevée à la cuisse d'une Brebis Texel; Mèche du milieu et Mèche inférieure: prélevées au flanc de deux Brebis 'Texel différentes.

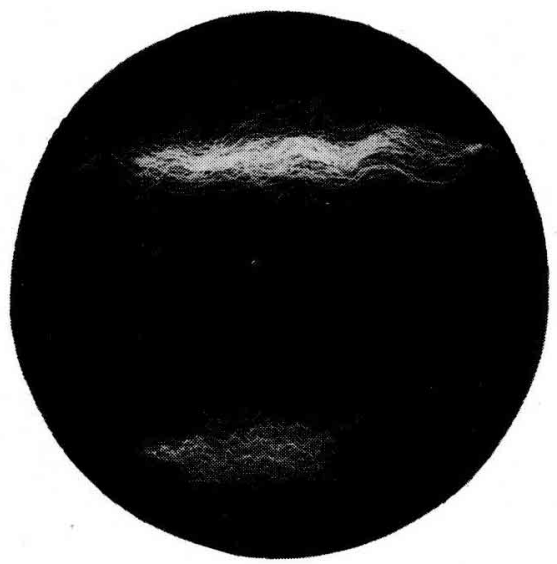

FIG. 2. - Photographie des mêmes mèches après immersion : Mèche supérieuve : Elle demeure visible car presque toutes les fibres ont des canaux médullaires s'étendant sur toute la longueur. Ces fibres sont des poils. Mèche du milieu : Elle est devenue invisible car toutes ses fibres sont des brins de laine. Mèche inférieure : Lécrèrement visible, car seules quelques fibres ont des canaux médullaires. Ces fibres sont des hétérotypes. 